\title{
ERROR BOUNDS FOR THE METHOD OF GOOD LATTICE POINTS
}

\author{
SHAUN DISNEY AND IAN H. SLOAN
}

\begin{abstract}
New error bounds are obtained for the method of good lattice points for multidimensional quadrature, when $m$, the number of quadrature points, is prime. One of these bounds reduces the constant in Niederreiter's asymptotic error bound, if the dimension exceeds 2 . Together they give very much smaller numerical bounds for all values of $m$.
\end{abstract}

\section{INTRODUCTION}

The method of good lattice points, developed by Korobov [4] and Hlawka [3], is a well-studied method for the approximate evaluation of integrals over the $s$-dimensional unit cube $I^{s}=[0,1]^{s}$, under the assumption that the integrand is 1-periodic in each variable. The method is reviewed by Niederreiter [7, 9].

If $f$ is such an integrand defined on $\mathbb{R}^{s}$, then the approximation is

$$
\int_{I^{5}} f(\mathbf{x}) d \mathbf{x} \approx \frac{1}{m} \sum_{j=0}^{m-1} f\left(\frac{j}{m} \mathbf{g}\right),
$$

where $m \geq 2$ is a (large) positive integer, and $\mathbf{g} \in \mathbb{Z}^{s}$ is an appropriate $s$ dimensional integer vector, or "lattice point". In the present work, as in the work of Korobov and Hlawka, $m$ is taken to be prime.

If $f$ has the absolutely convergent Fourier series expansion

$$
f(\mathbf{x})=\sum_{\mathbf{h} \in \mathbb{Z}^{s}} a_{\mathbf{h}} e^{2 \pi i \mathbf{h} \cdot \mathbf{x}},
$$

then, as is well known, the error in (1.1) is

$$
\frac{1}{m} \sum_{j=0}^{m-1} f\left(\frac{j}{m} \mathbf{g}\right)-\int_{I^{s}} f(\mathbf{x}) d \mathbf{x}=\sum_{\substack{\mathbf{h} \neq \mathbf{0} \\ \mathbf{h} \cdot \mathbf{g} \equiv 0(\bmod m)}} a_{\mathbf{h}} .
$$

Let

$$
r(\mathbf{h})=\prod_{i=1}^{s} r\left(h_{i}\right), \quad r(h)=\max (1,|h|) .
$$

Received May 15, 1989.

1980 Mathematics Subject Classification (1985 Revision). Primary 65D32. 
From (1.3) it follows that the least upper bound of the error for the class of functions whose Fourier coefficients satisfy $\left|a_{\mathbf{h}}\right| \leq r(\mathbf{h})^{-\alpha \gamma}$ for $\mathbf{h} \neq \mathbf{0}$ is

$$
P_{\alpha}(\mathbf{g}, m)=\sum_{\substack{\mathbf{h} \neq \mathbf{0} \\ \mathbf{h} \cdot \mathbf{g} \equiv 0(\bmod m)}} \frac{1}{r(\mathbf{h})^{(\alpha}}, \quad \alpha>1 .
$$

In the method of good lattice points, for fixed $m$ and $\alpha$ one chooses a lattice point $\mathbf{g}$ which makes $P_{\alpha}(\mathbf{g}, m)$ as small as possible. (Alternative selection criteria are discussed by Lyness [5].) A result of Niederreiter (obtained by combining (4.6) of [7] with Theorem 2 of [8]) is that for $m$ prime (or a prime power) there exists a lattice point $\mathbf{g}$ such that

$$
P_{\alpha}(\mathbf{g}, m)<(1+2 \zeta(\alpha))^{s} \frac{(2 \log m+0.81)^{s \alpha}+1}{m^{\alpha}}=O\left(\frac{(\log m)^{s \alpha}}{m^{\alpha}}\right) .
$$

For $m$ prime, Bakhvalov [1] has even shown that a bound of order

$$
O\left((\log m)^{(s-1) r} / m^{\alpha}\right)
$$

is achievable, but he does not give the constants.

In the present work we give new upper bounds on $P_{\alpha}(\mathbf{g}, m)$ for good choices of $\mathbf{g}$. One of these (see Theorem 5) is of the same asymptotic order as (1.6), but has a smaller constant factor in front, except possibly for $s=2$.

The main results are stated in the next section, and proved in $\$ 3$. Some of the results depend on making an appropriate choice of a certain parameter $\beta$. Motivations for our particular choices are given in $\S 4$. Finally, in $\S 5$ we calculate numerical values for the various bounds, and compare them with known "good" values of $P_{\alpha}(\mathbf{g}, m)$.

\section{THE MAIN RESUlts}

Our first result makes use of the mean of $P_{r r}(\mathbf{g}, m)$,

$$
M_{c r}(m)=\frac{1}{(m-1)^{s}} \sum_{\mathbf{g} \in G} P_{c}(\mathbf{g}, m), \quad \alpha>1,
$$

where $G$ is the set of all lattice points $\mathbf{g}=\left(g_{1}, g_{2}, \ldots, g_{s}\right)$ satisfying $-m / 2<$ $g_{j} \leq m / 2$ and $g_{j} \neq 0$ for $j=1, \ldots, s$.

Theorem 1. If $m$ is prime, then

$$
M_{c \gamma}(m)=\frac{(1+2 \zeta(\alpha))^{s}}{m}+\frac{(m-1)}{m}\left(1-\frac{2\left(1-m^{1-\alpha}\right) \zeta(\alpha)}{m-1}\right)^{s}-1 .
$$

This is proved in $\S 3$, using techniques adapted from Niederreiter [8].

Since $M_{r}(m)$ is the mean of $P_{r r}(\mathbf{g}, m)$ over $\mathbf{g}$, it is obvious that there exists a point $\mathbf{g}$ for which $P_{c r}(\mathbf{g}, m)$ is less than or equal to the mean. 
Corollary 2. If $m$ is prime, then there exists a lattice point $\mathbf{g}$ such that

$$
P_{\alpha}(\mathbf{g}, m) \leq \frac{(1+2 \zeta(\alpha))^{s}}{m}+\frac{(m-1)}{m}\left(1-\frac{2\left(1-m^{1-\alpha}\right) \zeta(\alpha)}{m-1}\right)^{s}-1 .
$$

If $m \geq \zeta(\alpha)+1$, then an obvious inequality is

$$
1>1-\frac{2\left(1-m^{1-\alpha}\right) \zeta(\alpha)}{m-1}>1-\frac{2 \zeta(\alpha)}{m-1} \geq-1 .
$$

This leads to the following corollary, slightly weaker, but more transparent than the results above:

Corollary 3. If $m$ is prime and $m \geq \zeta(\alpha)+1$, then

$$
M_{\alpha}(m) \leq(1+2 \zeta(\alpha))^{s} / m,
$$

and there exists a lattice point $\mathbf{g}$ such that

$$
P_{\alpha}(\mathbf{g}, m) \leq(1+2 \zeta(\alpha))^{s} / m .
$$

The bound in Corollary 2 is only of order $O(1 / \mathrm{m})$ and therefore is worse than the bound (1.6) for large enough values of $m$. However, we shall see in $\S 5$ that its numerical values are useful for small and moderate values of $m$, and indeed are smaller than the bound (1.6) for all practical values of $m$.

Bounds with better asymptotic form may now be generated by a simple application of Jensen's inequality (see Hardy et al. [2, Theorem 19])

$$
\left(\sum\left|a_{i}\right|^{p}\right)^{1 / p} \leq\left(\sum\left|a_{i}\right|^{q}\right)^{1 / q}, \quad 0<q<p
$$

which implies, by (1.5),

$$
P_{\alpha}(\mathbf{g}, m) \leq\left(P_{\beta}(\mathbf{g}, m)\right)^{\alpha / \beta}, \quad 1<\beta<\alpha .
$$

Combined with Theorem 1, this yields the following result:

Theorem 4. If $m$ is prime and $1<\beta<\alpha$, then there exists a lattice point $\mathbf{g}$ such that

$$
\begin{aligned}
P_{\alpha}(\mathbf{g}, m) & \leq\left(M_{\beta}(m)\right)^{\alpha / \beta} \\
& =\left(\frac{(1+2 \zeta(\beta))^{s}}{m}+\frac{(m-1)}{m}\left(1-\frac{2\left(1-m^{1-\beta}\right) \zeta(\beta)}{m-1}\right)^{s}-1\right)^{\alpha / \beta} .
\end{aligned}
$$

In principle, the best choice of $\beta$ in Theorem 4 is that which, for given $m$ and $\alpha$, minimizes $\left(M_{\beta}(m)\right)^{\alpha / \beta}$. In $\S 4$ we provide some motivation for two nonoptimal choices,

$$
\beta_{1}(m)=\log m /(\log m-s)=1+(\log \sqrt[s]{m}-1)^{-1},
$$

and

$$
\beta_{2}(m)=1+(\log (\sqrt[3]{m} / b)-\log \log (\sqrt[4]{m} / b))^{-1},
$$


where

$$
b=2 e^{-\gamma+1 / 2}
$$

and $\gamma=0.57721 \ldots$ is Euler's constant. The second of these is the better in both theory and practice, and is the one we use in association with (2.7) for obtaining numerical bounds in $\S 5$. The first choice $\beta_{1}$, on the other hand, is analytically simpler and allows us to obtain in $\S 3$ the following explicit bound:

Theorem 5. If $m$ is prime and $m>e^{s \alpha /(\alpha-1)}$, then there exists a lattice point $\mathbf{g}$ such that

$$
P_{\alpha}(\mathbf{g}, m) \leq\left(\frac{e}{s}\right)^{s \alpha} \frac{(2 \log m+s)^{s \alpha}}{m^{\alpha}} .
$$

The estimate $(2.11)$ has the asymptotic form $C(\log m)^{s \alpha} / m^{\alpha}$, where $C=(2 e / s)^{s \alpha}$. Niederreiter's estimate (1.6) has the same asymptotic form $C^{\prime}(\log m)^{s \alpha} / m^{\alpha}$, with the different constant factor $C^{\prime}=2^{s \alpha}(1+2 \zeta(\alpha))^{s}$. The ratio

$$
\frac{C^{\prime}}{C}=\left(\left(\frac{s}{e}\right)^{\alpha}(1+2 \zeta(\alpha))\right)^{s}
$$

is greater than 1 for $\alpha \leq 2$ and $s \geq 1$, and for all $\alpha$ if $s \geq 3$; and for fixed $\alpha$ it increases faster than exponentially with $s$. The larger bounds given by (1.6) are reflected in the numerical bounds computed in $\S 5$.

\section{Proofs}

3.1. Proof of Theorem 1. Since $M_{\alpha}(m)$ is the mean of $P_{\alpha}(\mathbf{g}, m)$, all that has to be proved is the explicit expression for $M_{\alpha}(m)$. In this proof, summations over $\mathbf{h}$ and $h$ will be over $\mathbb{Z}^{s}$ and $\mathbb{Z}$, unless specifically restricted. The notation $\sum^{*}$ stands for summation excluding zero.

We have

$$
\begin{aligned}
M_{\alpha}(m) & \left.=\frac{1}{(m-1)^{s}} \sum_{\mathbf{g} \in G} \sum_{\substack{\mathbf{h} \neq \mathbf{0} \\
\mathbf{h} \cdot \mathbf{g} \equiv 0}} \frac{1}{r(\mathbf{h o d})^{\alpha}}\right) \\
& =\frac{1}{(m-1)^{s}} \sum_{\mathbf{h}} N(\mathbf{h}) \frac{1}{r(\mathbf{h})^{\alpha}}-1,
\end{aligned}
$$

where $N(\mathbf{h})$ is the number of vectors $\mathbf{g} \in G$ such that $\mathbf{h} \cdot \mathbf{g} \equiv 0(\bmod m)$.

We can express $N(\mathbf{h})$ as

$$
N(\mathbf{h})=\sum_{\mathbf{g} \in G} \frac{1}{m} \sum_{j=0}^{m-1} e\left(\frac{j}{m} \mathbf{h} \cdot \mathbf{g}\right),
$$


where $e(t)$ denotes $e^{2 \pi i t}$. Then we have

$$
\begin{aligned}
M_{\alpha}(m)= & \frac{1}{m(m-1)^{s}} \sum_{j=0}^{m-1} \sum_{\mathbf{h}} \sum_{\mathbf{g} \in G} e\left(\frac{j}{m} \mathbf{h} \cdot \mathbf{g}\right) \frac{1}{r(\mathbf{h})^{\alpha}}-1 \\
= & \frac{1}{m(m-1)^{s}} \sum_{j=0}^{m-1} \sum_{h_{1}=-\infty}^{\infty} \cdots \sum_{h_{s}=-\infty}^{\infty} \\
& \sum^{*} \ldots \sum_{-m / 2<g_{1} \leq m / 2}^{*} \frac{e\left((j / m) h_{1} g_{1}\right) \cdots e\left((j / m) h_{s} g_{s}\right)}{r\left(h_{1}\right)^{\alpha} \cdots r\left(h_{s}\right)^{\alpha}}-1 \\
= & \frac{1}{m} \sum_{j=0}^{m-1}\left(\frac{1}{m-1} \sum_{h-m / 2} \sum_{m<g \leq m / 2}^{*} \frac{e((j / m) h g)}{r(h)^{\alpha}}\right)^{s}-1 .
\end{aligned}
$$

Separating out the $j=0$ term, we get

$$
M_{\alpha}(m)=\frac{1}{m}(1+2 \zeta(\alpha))^{s}+\frac{1}{m} \sum_{j=1}^{m-1}\left(\frac{1}{m-1} T(j)\right)^{s}-1,
$$

where

$$
T(j)=\sum_{h-m / 2<g \leq m / 2} \sum^{*} e\left(\frac{j}{m} h g\right) \frac{1}{r(h)^{\alpha}}, \quad 1 \leq j \leq m-1 .
$$

Separating out the terms with $h \equiv 0(\bmod m)$, we have

$$
\begin{aligned}
T(j)= & \sum_{h} \frac{1}{r(h m)^{\alpha}} \sum_{-m / 2<g \leq m / 2}^{*} 1 \\
& +\sum_{h \neq 0(\bmod m)} \frac{1}{r(h)^{\alpha}} \sum_{-m / 2<g \leq m / 2}^{*} e\left(\frac{j}{m} h g\right) \\
= & (m-1) \sum_{h} \frac{1}{r(h m)^{\alpha}}-\sum_{h \not 0(\bmod m)} \frac{1}{r(h)^{\alpha}} \\
= & (m-1)\left(1+\frac{2}{m^{\alpha}} \zeta(\alpha)\right)-\left(\sum_{h \neq 0} \frac{1}{r(h)^{\alpha}}-\sum_{h}^{*} \frac{1}{r(h m)^{\alpha}}\right) \\
= & (m-1)\left(1+\frac{2}{m^{\alpha}} \zeta(\alpha)\right)-2\left(\zeta(\alpha)-\frac{1}{m^{\alpha}} \zeta(\alpha)\right) \\
= & (m-1)-2\left(1-m^{1-\alpha}\right) \zeta(\alpha) .
\end{aligned}
$$

Putting this into (3.1) gives (2.2).

3.2. Proof of Theorem 5. If $m \geq 1, s \geq 1$, then

$$
m \geq \frac{1}{s} \log m+1=\frac{1}{\beta_{1}(m)-1}+2,
$$

which, together with the inequality

$$
\zeta(t)=\sum_{1}^{\infty} \frac{1}{n^{t}}<1+\int_{1}^{\infty} \frac{1}{x^{t}} d t=\frac{1}{t-1}+1, \quad t>1,
$$


implies that $m>\zeta\left(\beta_{1}(m)\right)+1$ for all $m \geq 1, s \geq 1$, where $\beta_{1}(m)$ is given by (2.8). It follows that Corollary 3 is applicable with $\alpha$ replaced by $\beta_{1}(m)$, giving

$$
M_{\beta_{1}(m)}(m) \leq\left(1+2 \zeta\left(\beta_{1}(m)\right)\right)^{s} / m .
$$

The assumption $m>e^{s \alpha /(\alpha-1)}$ is equivalent to $1<\beta_{1}(m)<\alpha$, so Theorem 4 asserts the existence of a $\mathbf{g}$ such that

$$
\begin{aligned}
P_{\alpha}(\mathbf{g}, m) & \leq\left(\frac{\left(1+2 \zeta\left(\beta_{1}(m)\right)\right)^{s}}{m}\right)^{\alpha / \beta_{1}(m)}<\frac{\left(2\left(\beta_{1}-1\right)^{-1}+3\right)^{s \alpha / \beta_{1}}}{m^{\alpha / \beta_{1}}} \\
& =\frac{(2 \log \sqrt[s]{m}+1)^{s \alpha / \beta_{1}}}{m^{\alpha} e^{-s \alpha}}<\frac{(2 \log \sqrt[s]{m}+1)^{s \alpha}}{m^{\alpha} e^{-s \alpha}} \\
& =\left(\frac{e}{s}\right)^{s \alpha} \frac{(2 \log m+s)^{s \alpha}}{m^{\alpha}} \cdot
\end{aligned}
$$

4. Motivation For Choosing $\beta=\beta_{1}$ AND $\beta=\beta_{2}$

We begin with a one-parameter family of choices for $\beta$ in Theorem 4, namely

$$
\beta=\log m /(\log m-c)=1+(\log \sqrt[c]{m}-1)^{-1}, \quad c>0 .
$$

Proposition 6. If $m$ is prime and $\beta=\beta(m)$ is defined by (4.1), then

$$
\left(M_{\beta}(m)\right)^{\alpha / \beta} \sim\left(\frac{2^{s} e^{c}}{c^{s}}\right)^{\alpha} \frac{(\log m)^{s \alpha}}{m^{\alpha}} \text { as } m \rightarrow \infty .
$$

Proof. Using $\beta \rightarrow 1$ as $m \rightarrow \infty$ and $\zeta(t)=(t-1)^{-1}+O(1)$ as $t \rightarrow 1$ (for the latter see Whittaker and Watson $[10, \S 13.21])$, we obtain

$$
\zeta(\beta)=(\beta-1)^{-1}+O(1)=\log \sqrt[c]{m}+O(1),
$$

and hence, using (2.2),

$$
M_{\beta}=\frac{(2 \log \sqrt[c]{m})^{s}}{m}+O\left(\frac{(\log m)^{s-1}}{m}\right) .
$$

Since $1 / \beta=1-c / \log m$ and $m^{-c / \log m}=e^{-c}$, the result (4.2) follows.

It is now reasonable to choose the parameter $c$ in (4.1) so as to minimize the constant factor in the asymptotic expression (4.2). Elementary calculus shows that $e^{c} / c^{s}$ is minimized by the choice $c=s$. With this choice (4.1) yields $\beta=\beta_{1}$.

The choice $\beta=\beta_{2}$ does not improve the asymptotic expression for $\left(M_{\beta}\right)^{\alpha / \beta}$ : in fact, it can be shown that the choices $\beta_{1}$ and $\beta_{2}$ both yield the same asymptotic form,

$$
\left(M_{\beta_{i}}(m)\right)^{\alpha / \beta_{l}} \sim\left(\frac{2 e}{s}\right)^{s \alpha} \frac{(\log m)^{s \alpha}}{m^{\alpha}}, \quad i=1,2
$$


The motivation for choosing $\beta=\beta_{2}$ comes from the following more careful argument. Let $F(\beta, m)$ be the bound in Theorem 4, i.e.,

$$
F(\beta, m)=\left(M_{\beta}(m)\right)^{\alpha / \beta} \text {. }
$$

Then

$$
\begin{aligned}
\frac{\partial F}{\partial \beta}(\beta, m) & =F(\beta, m)\left(-\frac{\alpha}{\beta^{2}} \log M_{\beta}+\frac{\alpha}{\beta M_{\beta}} \frac{\partial M_{\beta}}{\partial \beta}\right) \\
& =-\frac{\alpha}{\beta^{2}} F(\beta, m) \log (H(\beta, m)),
\end{aligned}
$$

where

$$
H(\beta, m)=M_{\beta}(m) \exp \left(-\frac{\beta}{M_{\beta}(m)} \frac{\partial M_{\beta}}{\partial \beta}(m)\right) .
$$

Thus, $\partial F / \partial \beta=0$ if and only if $H(\beta, m)=1$; and it can be shown that the stationary value of $F$ is in fact a minimum. The next proposition shows that the choice $\beta=\beta_{2}$ is in a certain sense asymptotically optimal, in that $H\left(\beta_{2}(m), m\right) \rightarrow 1$ as $m \rightarrow \infty$, whereas this is not true for the choice $\beta=\beta_{1}$.

Proposition 7. If $m$ is prime and $s \geq 3$, then

(i) $H\left(\beta_{1}(m), m\right) \rightarrow+\infty$ as $m \rightarrow \infty$,

(ii) $H\left(\beta_{2}(m), m\right) \rightarrow 1$ as $m \rightarrow \infty$.

Proof. Let $\zeta_{i}=\zeta\left(\beta_{i}(m)\right), \zeta_{i}^{\prime}=\zeta^{\prime}\left(\beta_{i}(m)\right)$, and $x_{i}=\left(\beta_{i}(m)-1\right)^{-1}$ for $i=$ 1,2. Then $x_{1}=\log \sqrt[5]{m}-1$ and $x_{2}=\log (\sqrt[5]{m} / b)-\log \log (\sqrt[5]{m} / b)$, and in both cases $x_{i}=\log \sqrt[s]{m}+O(\log \log m)$ as $m \rightarrow \infty$. Since

$$
\zeta(t)=(t-1)^{-1}+\gamma+\varepsilon(t),
$$

where $\varepsilon(t) \rightarrow 0$ as $t \rightarrow 1$, we have

$$
\begin{gathered}
\zeta_{i}=x_{i}+O(1) \quad \text { as } m \rightarrow \infty, \quad i=1,2, \\
1+2 \zeta_{i}=2 x_{i}\left(1+\frac{2 \gamma+1}{2 x_{i}}+o\left(\frac{1}{x_{i}}\right)\right) \quad \text { as } m \rightarrow \infty, i=1,2 .
\end{gathered}
$$

Also $\zeta^{\prime}(t)=-(t-1)^{-2}+O(1)$, which implies

We also require

$$
\zeta_{i}^{\prime}=-x_{i}^{2}+O(1) \text { as } m \rightarrow \infty
$$

$$
m^{1-\beta_{i}(m)}=m^{-1 / x_{i}}=m^{-1 /(\log \sqrt[s]{m}+O(\log \log m))}=e^{-s}(1+o(1)) .
$$

From (2.2) and (4.8) we have

$$
\begin{aligned}
M_{\beta_{i}(m)}(m)= & \frac{1}{m}\left(1+2 \zeta_{i}\right)^{s}-\frac{1}{m}\left(1+2\left(1-e^{-s}(1+o(1))\right) s \zeta_{i}\right) \\
& +O\left((\log m)^{2} / m^{2}\right) \\
= & \frac{1}{m}\left(\left(1+2 \zeta_{i}\right)^{s}-\left(1+2\left(1-e^{-s}\right) s \zeta_{i}\right)(1+o(1))\right) \\
= & \frac{1}{m}\left(1+2 \zeta_{i}\right)^{s}\left(1+O\left(1 / x_{i}^{2}\right)\right),
\end{aligned}
$$


since $s \geq 3$. Similarly, by differentiating (2.2) and applying (4.6) and (4.7) we obtain

$$
\left.\frac{\partial M_{\beta}(m)}{\partial \beta}\right|_{\beta=\beta_{l}(m)}=-\frac{2 s}{m}\left(1+2 \zeta_{i}\right)^{s-1} x_{i}^{2}\left(1+O\left(1 / x_{i}^{2}\right)\right) .
$$

Thus, the argument of the exponential function in the expression (4.5) for $H(\beta, m)$ is

$$
\begin{aligned}
\left.\frac{-\beta_{i}(m)}{M_{\beta_{i}}(m)} \frac{\partial M_{\beta}(m)}{\partial \beta}\right|_{\beta=\beta_{i}} & =\frac{\left(1+1 / x_{i}\right) 2 s x_{i}^{2}\left(1+O\left(1 / x_{i}^{2}\right)\right)}{\left(1+2 \zeta_{i}\right)\left(1+O\left(1 / x_{i}^{2}\right)\right)} \\
& =\frac{s\left(x_{i}^{2}+x_{i}+O(1)\right)}{x_{i}\left(1+(2 \gamma+1) / 2 x_{i}+o\left(1 / x_{i}\right)\right)} \\
& =s\left(x_{i}+1+O\left(\frac{1}{x_{i}}\right)\right)\left(1-\frac{2 \gamma+1}{2 x_{i}}+o\left(\frac{1}{x_{i}}\right)\right) \\
& =s\left(x_{i}+\frac{1-2 \gamma}{2}+o(1)\right) .
\end{aligned}
$$

Using (4.9) and (4.11), it now follows from the definition (4.5) of $H(\beta, m)$ that

$$
H\left(\beta_{i}(m), m\right)^{1 / s}=\frac{1}{\sqrt[3]{m}}\left(2 x_{i}+O(1)\right) \exp \left(x_{i}+\frac{1-2 \gamma}{2}+o(1)\right), \quad i=1,2 .
$$

In particular,

$$
\begin{aligned}
H\left(\beta_{1}(m), m\right)^{1 / s} & =(2 \log \sqrt[5]{m}+O(1)) \exp \left(\frac{-1-2 \gamma}{2}+o(1)\right) \\
& \rightarrow+\infty \text { as } m \rightarrow \infty,
\end{aligned}
$$

whereas

$$
\begin{aligned}
H\left(\beta_{2}(m), m\right)^{1 / s}= & \left(2 \log \frac{\sqrt[3]{m}}{b}-2 \log \log \frac{\sqrt[3]{m}}{b}+O(1)\right) \\
& \times \frac{1}{b} \exp \left(-\log \log \frac{\sqrt[4]{m}}{b}+\frac{1-2 \gamma}{2}+o(1)\right) \\
= & 2 \log \frac{\sqrt[3]{m}}{b}(1+o(1)) \frac{1}{b}\left(\log \frac{\sqrt[1]{m}}{b}\right)^{-1} \exp \left(\frac{1-2 \gamma}{2}+o(1)\right) \\
\rightarrow & \frac{2}{b} \exp \left(\frac{1-2 \gamma}{2}\right)=1 \text { as } m \rightarrow \infty .
\end{aligned}
$$

\section{Numerical estimates}

In Tables 1, 2, and 3 we show numerical values of theoretical bounds for the case $\alpha=2$ and dimensions $s=3,6$, and 10 . The bounds are calculated from Theorem 1, from Theorem 4 with $\beta=\beta_{2}(m)$ (see (2.9)), and from Niederreiter's bound (1.6). Additionally, to give some perspective on what might be 
achievable, we show some known "good" values of $P_{2}(\mathbf{g}, m)$ for comparable values of $m$. These are taken from the tables of Maisonneuve [6].

The tables show, perhaps surprisingly, that the bound given by Corollary 2 is a quite effective bound for all practical values of $m$, notwithstanding its inferior asymptotic behavior. The bound given by Theorem 4 with $\beta=\beta_{2}(m)$, though asymptotically better, produces a smaller bound only for values of $m$ exceeding about $10^{s+1}$, and even then makes only a modest improvement.

The bound given by (1.6) exceeds both of the other bounds by many orders of magnitude, especially when $s$ is large, and is therefore less useful as a numerical estimate.

\section{TABLE 1}

Bounds on $P_{2}$ for $s=3$

\begin{tabular}{c|ccl|crl}
\hline$m$ & Cor. 2 & $\begin{array}{c}\text { Thm. 4 } \\
\left(\beta=\beta_{2}\right)\end{array}$ & $\begin{array}{c}\text { Bound } \\
\text { of }(1.6)\end{array}$ & $\begin{array}{c}\text { "Good" values } \\
\text { of } P_{2}(\mathbf{g}, m)\end{array}$ & $m$ & $\mathbf{g}$ \\
\hline $10^{2}$ & $6.8(-1)$ & $6.9(-1)$ & $8.0(3)$ & $2.2(-1)$ & 98 & $(1,16,44)$ \\
$10^{3}$ & $6.8(-2)$ & $7.1(-2)$ & $7.2(2)$ & $5.3(-3)$ & 1010 & $(1,140,237)$ \\
$10^{4}$ & $6.8(-3)$ & $5.4(-3)$ & $3.9(1)$ & $1.3(-4)$ & 10,007 & $(1,544, \ldots)$ \\
$10^{5}$ & $6.8(-4)$ & $2.9(-4)$ & 1.4 & $4.9(-6)$ & 100,063 & $(1,53584, \ldots)$ \\
$10^{6}$ & $6.8(-5)$ & $1.2(-5)$ & $4.1(-2)$ & & & \\
\hline
\end{tabular}

TABLE 2

Bounds on $P_{2}$ for $s=6$

\begin{tabular}{|c|c|c|c|c|c|c|}
\hline$m$ & Cor. 2 & $\begin{array}{l}\text { Thm. } 4 \\
\left(\beta=\beta_{2}\right)\end{array}$ & $\begin{array}{c}\text { Bound } \\
\text { of }(1.6)\end{array}$ & $\begin{array}{l}\text { "Good" values } \\
\text { of } P_{2}(\mathbf{g}, \mathrm{m})\end{array}$ & $m$ & g \\
\hline $10^{3}$ & 6.2 & $1.1(1)$ & $6.0(11)$ & & & \\
\hline $10^{4}$ & $6.2(-1)$ & $6.3(-1)$ & $1.6(11)$ & $2.9(-1)$ & 10,007 & $(1,2240, \ldots)$ \\
\hline $10^{5}$ & $6.2(-2)$ & $6.5(-2)$ & $2.1(10)$ & $1.8(-2)$ & 100,063 & $(1,43307, \ldots)$ \\
\hline $10^{6}$ & $6.2(-3)$ & $6.6(-3)$ & $1.7(9)$ & & & \\
\hline $10^{7}$ & $6.2(-4)$ & $5.6(-4)$ & $1.1(8)$ & & & \\
\hline
\end{tabular}

TABLE 3

Bounds on $P_{2}$ for $s=10$

\begin{tabular}{c|lll|lll}
\hline$m$ & Cor. 2 & $\begin{array}{c}\text { Thm. 4 } \\
\left(\beta=\beta_{2}\right)\end{array}$ & $\begin{array}{c}\text { Bound } \\
\text { of }(1.6)\end{array}$ & $\begin{array}{c}\text { "Good" values } \\
\text { of } P_{2}(\mathbf{g}, m)\end{array}$ & $m$ & $\mathbf{g}$ \\
\hline $10^{4}$ & $2.1(2)$ & $6.4(3)$ & $1.0(24)$ & & & \\
$10^{5}$ & $2.1(1)$ & $5.5(1)$ & $7.4(23)$ & $2.1(1)$ & 103,661 & $(1,45681, \ldots)$ \\
$10^{6}$ & 2.1 & 2.5 & $2.5(23)$ & & & \\
$10^{7}$ & $2.1(-1)$ & $2.1(-1)$ & $5.1(22)$ & & & \\
$10^{8}$ & $2.1(-2)$ & $2.2(-2)$ & $6.9(21)$ & & & \\
\hline
\end{tabular}




\section{BIBLIOGRAPHY}

1. N. S. Bakhvalov, Approximate computations of multiple integrals, Vestnik Moskov. Univ. Ser. Mat. Mekh. Astr. Fiz. Him. 4 (1959), 3-18. (Russian)

2. G. H. Hardy, J. E. Littlewood. and G. Pólya, Inequalities, University Press, Cambridge, 1934.

3. E. Hlawka, Zur angenäherten Berechnung mehrfacher Integrale, Monatsh. Math. 66 (1962), 140-151.

4. N. M. Korobov, The approximate computation of multiple integrals, Dokl. Akad. Nauk SSSR 124 (1959), 1207-1210. (Russian)

5. J. N. Lyness, Some comments on quadrature rule construction criteria, Numerical Integration. III (H. Brass and G. Hämmerlin, eds.), ISNM 85, Birkhäuser, Basel, 1988, pp. 117-129.

6. D. Maisonneuve, Recherche et utilisation des 'Bons Treillis'. Programmation et résultats numériques, Applications of Number Theory to Numerical Analysis (S. K. Zaremba, ed.), Academic Press, New York, 1972, pp. 121-201.

7. H. Niederreiter, Quasi-Monte Carlo methods and pseudo-random numbers, Bull. Amer. Math. Soc. 84 (1978), 957-1041.

8. __ Existence of good lattice points in the sense of Hlawka, Monatsh. Math. 86 (1978), 203-219.

9. __ Quasi-Monte Carlo methods for numerical integration, Numerical Integration. III (H. Brass and G. Hämmerlin, eds.), ISNM 85, Birkhäuser, Basel, 1988, pp. 157-171.

10. E. T. Whittaker and G. N. Watson, A course on modern analysis, 4 th ed., University Press, Cambridge, 1927.

School of Mathematics, University of New South Wales, Sydney, New South Wales 2033, Australia

E-mail address: sloan@hydra.maths.unsw.oz.au 